\title{
Novel Self-loop basis functions for the stability of the Linear-linear discretization of the Electric Field Integral Equation at very low frequencies
}

\author{
Jose M. Tamayo *, Eduard Ubeda and Juan M. Rius \\ Technical University of Catalonia (UPC), Dept. of Signal Theory and \\ Communications (TSC), Barcelona, Spain
}

\section{Introduction}

The Linear-linear (LL) basis functions [1] stand for a divergence-conforming set with linear expansion of both normal and tangential directions of the normal component of the electric current across the edge. Recently, this set has received special attention because it reduces the observed discrepancy in the RCS computation with the RWG [2] discretization in Method of Moments of the Magnetic Field Integral Equation (MFIE) [3]. The LL-discretization of the Electric-field Integral Equation (EFIE) is required in combined formulations that benefit from the MFIE error reduction, such as, for example, the Combined Field Integral Equation (CFIE) [3] or the PMCHWT formulation [4], in the analysis, respectively, of perfectly conducting or penetrable scatterers. These formulations suffer from the EFIE low-frequency breakdown. At very low frequencies, the contribution of the vector potential to the impedance matrix becomes negligible, according to the finite machine precision, compared with the scalar potential. This makes the discretization of the EFIE ill-conditioned and the solution inaccurate. For double machine precision, the low-frequency breakdown appears when analyzing objects with sizes of the mesh cells below $10^{-8} \lambda$ [5]

The Loop-Tree or Loop-Star [6] basis functions overcome the low-frequency breakdown arising from the RWG-discretization of the EFIE. The RWG basis functions, with constant expansion along the tangential direction of the normal component of the current, are complete to 0 -order in the expansion of the divergence. Even though the LL basis functions double the number of unknowns, they remain complete to 0-order in the charge expansion. Therefore, in this context, the generalized Loop-Tree decomposition schemes for MoMdiscretizations complete to higher divergence-orders [7] are not required.

In this paper, we present a new scheme to obtain a stable discretization in Method of Moments of the EFIE with the LL-discretization in the very low frequency regime to overcome the low-frequency breakdown. Our strategy is based upon combining a novel set of basis functions, the Self-Loop basis functions, with the well-known Loop-Star basis functions.

\section{Self-Loop basis functions}

The RWG basis functions result from the addition of the First-kind (LL-1) and the Second-kind (LL-2) Linear-linear basis functions (see Fig. 1). We define the Self- 
Loop basis functions as the subtraction of the LL-1 and LL-2 basis functions. In consequence, the LL-1 and LL-2 basis functions can be decomposed, in view of the definition above, as the addition and the subtraction, respectively, of the RWG and the Self-Loop basis functions.

RWG

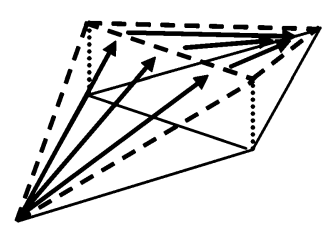

LL-1

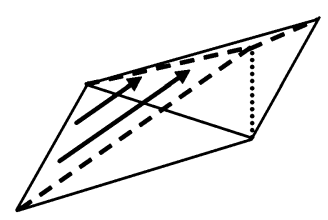

LL-2

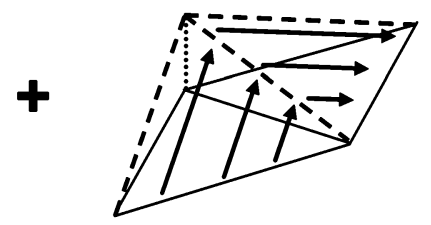

Fig. 1 Decomposition of the RWG basis functions in terms of the First-kind and Second-kind Linear-linear basis functions

Since the Self-Loop basis functions have zero divergence, they expand a solenoidal space where the degrees of freedom coincide with the number of interior edges arising from the discretization. Therefore, the RWG and the LL discretizations expand the same non-solenoidal space.

The strategies to yield a stable matrix system at very low frequencies are based on the rearrangement of the original basis functions into their solenoidal an nonsolenoidal subspaces [5][7]. The solenoidal and non-solenoidal subspaces arising from the RWG-discretization are expanded by the Loop and the Star subsets [5]. Similarly, the Loop and the Self-Loop basis functions expand the solenoidal subset arising from the LL-discretization, whereas the Star basis functions expand the remaining non-solenoidal part.

\section{Results}

We analyze a cube with side $0.1 \mathrm{~m}$ discretized with 192 triangular facets under an impinging x-polarized plane wave with $+z$-propagation. In Fig. 2, we show the condition number of the resulting impedance matrix for the discretizations EFIE[RWG], EFIE[LL], EFIE[Loop;Star], EFIE[Loop;Star;Self-Loop] and increasing wavelength values from $\lambda=1 \mathrm{~m}$ up to $\lambda=10^{15} \mathrm{~m}$. Since the EFIE gets unbalanced for very low decreasing frequencies because the vector and scalar potentials scale differently, we have frequency normalized the solenoidal and non-solenoidal contributions in the field equations and in the current expansion for the discretizations EFIE[Loop;Star] [8] and EFIE[Loop;Star;Self-Loop]. In view of Fig. 2, EFIE[LL] blows up at a slightly higher frequency than EFIE[RWG]. Moreover, the discretization with EFIE[Loop;Star;Self-Loop] produces a stable condition number, within the accuracy of the machine, in the same manner as EFIE[Loop;Star] all over the very low frequency range. In Fig. 3, we show the RCS computed with the discretizations EFIE[Loop;Star] and 
EFIE[Loop;Star;Self-loop] and $\lambda=10^{11} \mathrm{~m}$, where the other discretizations EFIE[RWG] and EFIE[LL] fail.

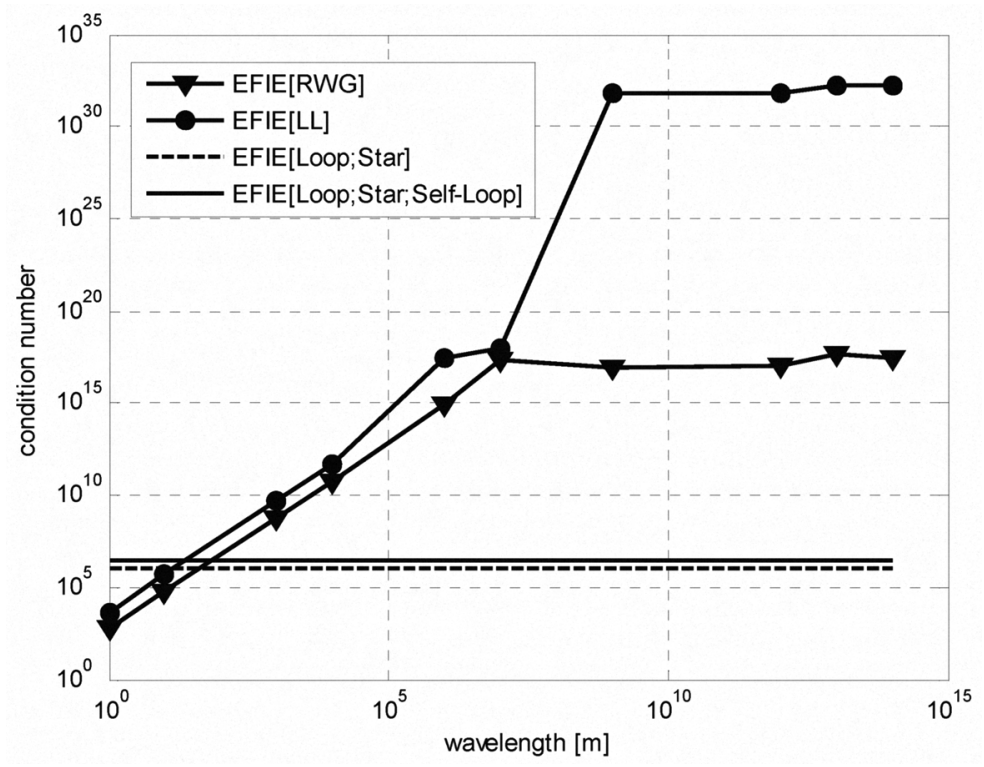

Fig.2 Condition number for growing values of the wavelength of the impedance matrix due to the following discretizations in Method of Moments of the EFIE:

[RWG], [LL], [Loop;Star] and [Loop;Star;Self-Loop]

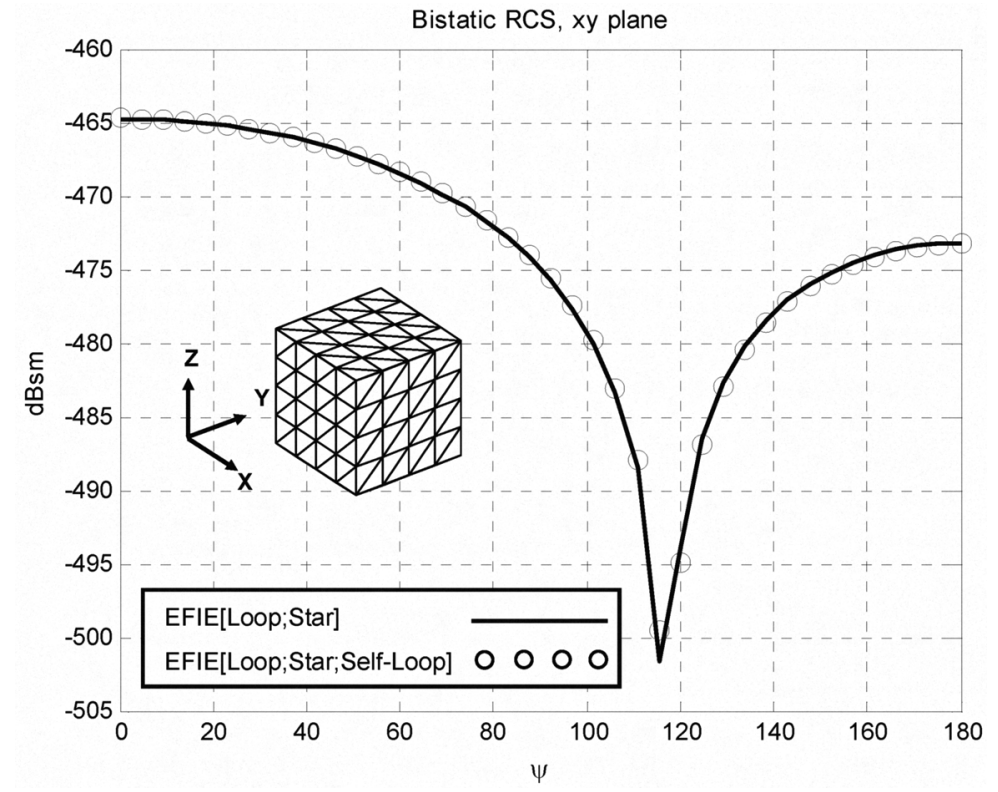

Fig. 3 Bistatic $x y$-plane for a cube with side $=0.1 \mathrm{~m}$ meshed with 192 triangles under an impinging plane wave with $\lambda=10^{11} \mathrm{~m}$ 


\section{Conclusions}

We present the Self-Loop basis functions, a new set of solenoidal basis functions, which, together with the Loop-Star basis functions, define a rearrangement of the LL-discretization in Method of Moments of the EFIE that results in a stable impedance matrix at very low frequencies.

\section{Acknowledgements}

This work was supported by the Spanish Interministerial Commission on Science and Technology (CICYT) under Projects TEC2006-13248-C04-02/TCM, TEC2007-66698-C04-01/TCM and CONSOLIDER CSD2008-00068 and by the

"Ministerio de Educación y Ciencia" through the FPU fellowship program.

\section{References}

[1] L. C. Trintinalia and H. Ling, "First order triangular patch basis functions for electromagnetic scattering analysis", J. Electromagn. Waves Appl., 15, 15211537,2001

[2] S. M. Rao, D. R. Wilton and A. W. Glisson, "Electromagnetic Scattering by surfaces of Arbitrary Shape", IEEE Transactions on Antennas and Propagation, vol. AP-30, No. 3, pp. 409-418, May 1982

[3] Ö. Ergül and L. Gürel, "Linear-Linear Basis functions for MLFMA solutions of magnetic-field and combined-field integral equations", IEEE Transactions on Antennas and Propagation, vol. 55, no. 4, April 2007

[4] P. Yla-Ölijala, M. Taskinen and S. Järvenpää, "Surface integral equation formulations for solving electromagnetic scattering methods with iterative methods", Radio Science, vol. 40, RS6002, doi: 10.1029/2004RS003169, 2005

[5] Z. G. Qian and W. C. Chew, "A quantitative study on the low frequency breakdown of the EFIE", Microwave and Optical Technology letters, vol. 50, no. 5, May 2008.

[6] W. Wu, A. W. Glisson amd D. Kajfez, "A study of two numerical procedures for the Electric Field Integral Equation at low frequency", Appl. Computat. Electromagn. Soc. J., vol. 10, no. 3, November 1995.

[7] R. A. Wildman and D. S. Weile, "An accurate Broad-band method of moments using higher basis functions and Tree-Loop decomposition", IEEE Transactions on Antennas and Propagation, vol. 52, no. 11, November 2004.

[8] J. Zhao and W. C. Chew, "Integral equation solution of Maxwell's equations from 0 frequency to microwave frequencies", IEEE Transactions on Antennas and Propagation, vol. 48, no. 10, pp. 1635-1645, October 2000 